\title{
Técnica de llizarov nas osteotomias do fêmur proximal e pélvica tripla para o tratamento da displasia do desenvolvimento do quadril em adolescentes*
}

\section{Ilizarov Technique with Proximal Femoral and Triple Pelvic Osteotomy for the Treatment of Adolescent Developmental Dysplasia of the Hip}

\author{
Mikhail Teplenky ${ }^{1}$ Waleed Mekki ${ }^{1}$ Evgenii Oleinikov ${ }^{1}$ \\ ${ }^{1}$ Departmento de Ortopedia Pediátrica No. 11, Centro Científico \\ Russo llizarov para Traumatologia Restauradora e Ortopedia, \\ Kurgan, Rússia \\ Rev Bras Ortop 2020;55(2):232-238.

\begin{abstract}
Endereço para correspondência Waleed Mekki, MBBS, MSC, MD, Department of Pediatric Orthopedics No. 11, Russian Ilizarov Scientific Center for Restorative Traumatology and Orthopedics, 6, M. Ulyanova Street, Kurgan, 640014, Russia
\end{abstract} \\ (e-mail: waleedmekki@excite.com).
}

\begin{abstract}
Resumo
Palavras-chave

- displasia do quadril

- osteoartrite do quadril

- osteotomia

- adolescente

Objetivo A importância das osteotomias pélvicas na coxartrose desenvolvida ainda é discutível. Alguns autores acreditam que a incongruência e a osteoartrite inicial das superfícies articulares são contraindicação para a cirurgia de preservação articular e estimularão maior progressão. A visão oposta é a de que a osteotomia pélvica tripla pode ser uma alternativa à substituição articular precoce. Este estudo relata resultados de médio a longo prazo de pacientes adolescentes com coxartrose desenvolvida, tratados por osteotomias proximais do fêmur e pélvica tripla, fixados pela técnica de llizarov.

Métodos Revisão retrospectiva entre 2002 e 2014 do tratamento de 26 pacientes com coxartrose devido a displasia do desenvolvimento do quadril. A amostra continha 22 mulheres e 4 homens. A idade média na operação foi de 14,7 anos (variação: 12-18 anos), e o acompanhamento médio foi de 5,9 anos (variação: 3-13).

Resultados Os resultados funcionais iniciais de acordo com o método de Merle d'Aubigné e Postel foram: dor $-4,3 \pm 0,05$ pontos; mobilidade $-3,6 \pm 0,3$ pontos; e marcha $-4 \pm 0,15$ pontos. O valor médio do índice da zona de carga (ZC) foi $38,7^{\circ} \pm 2.721^{\circ}$. O coeficiente acetabular (CA) foi de $162 \pm 6,8$, e o ângulo centro-borda (ACB) de Wiberg foi de $3^{\circ} \pm 0,2^{\circ}$. Os resultados foram acompanhados por 3 a 13 anos. No acompanhamento final, os resultados radiográficos mostraram que o valor do índice da ZC diminuiu para $8.2 \pm 1.293^{\circ}$ $\left(0^{\circ}-15^{\circ}\right)$, e o CA aumentou para $249 \pm 12,05$. A média do ângulo cervicodiafisário (ACD) foi $115^{\circ} \pm 4^{\circ}$, a distância articulotrocantérica (DAT) foi de $8,5 \pm 1,5 \mathrm{~mm}$, e o ACB de Wiberg foi $28^{\circ} \pm 1,6^{\circ}$ no acompanhamento final. A distribuição das articulações segundo Tönnis et al foi: grau I - 17 articulações; grau II - 8 articulações; e grau III - 1 articulação. O resultado foi bom em 14 pacientes (54\%), razoável em 10 pacientes (34,5\%), e ruim em 2 (11.5\%).
\end{abstract}

\footnotetext{
Estudo realizado no Departamento de Ortopedia Pediátrica do Centro Científico Russo Ilizarov para Traumatologia Restauradora e Ortopedia, Kurgan, Rússia.
}

recebido

25 de Outubro de 2018 aceito

05 de Fevereiro de 2019
DOI https://doi.org/

10.1055/s-0039-3400737. ISSN $0102-3616$.
Copyright $(2020$ by Sociedade Brasileira License terms de Ortopedia e Traumatologia. Published by Thieme Revinter Publicações Ltda, Rio de Janeiro, Brazil 


\begin{abstract}
Keywords

- hip dysplasia

- hip osteoarthritis

- osteotomy

- adolescent

Objective The significance of pelvic osteotomies in developed coxarthrosis is still disputable. Some authors believe that incongruency and early osteoarthritis of the articular surfaces are contraindications for joint-preserving surgery and will stimulate further progression. The opposite view is that triple pelvic osteotomy can be an alternative to early joint replacement. The present study reports the mid to long term results of adolescent patients with developed coxarthrosis treated by proximal femoral and triple pelvic osteotomies and fixed by the llizarov technique.

Methods A retrospective review between 2002 and 2014 of the treatment of 26 patients with coxarthrosis due to developmental dysplasia of the hip (DDH). The sample was composed of 22 female and 4 male subjects with a mean age at operation of 14.7 years (range: $12-18$ years) and mean follow-up of 5.9 years (range: 3-13 years) . Results The initial functional results according to the Merle d'Aubigné and Postel criteria were: pain $-4.3 \pm 0.05$ points; range of motion $-3.6 \pm 0.3$ points; and gait $4 \pm 0.15$ points. The average index of the weight bearing zone (WBZ) was of $38.7^{\circ} \pm 2.721^{\circ}$. The acetabular coefficient (AC) was of $162 \pm 6.8$, the center-edge angle (CEA) of Wiberg was of $3^{\circ} \pm 0.2^{\circ}$. The outcomes were followed up from 3 to 13 years. At the final follow-up, the radiographic outcomes showed that the value of the WBZ decreased to $8.2^{\circ} \pm 1.293^{\circ}\left(0-15^{\circ}\right)$, and that the $A C$ increased to $249 \pm 12.05$. The average neck-shaft angle (NSA) was of $115^{\circ} \pm 4^{\circ}$, the articulo-trochanteric distance (ATD) was of $8,5 \pm 1,5 \mathrm{~mm}$, and the CEA of Wiberg was of $28^{\circ} \pm 1.6^{\circ}$ ) at the final follow-up. The distribution of the joints according to Tönnis et al was: grade I 17 joints; grade II - 8 joints; and grade III - 1 joint. The outcomes were good for 14 patients (54\%), fair for 10 patients (34.5\%), and poor for 2 (11.5\%) patients.

Conclusion The treatment of adolescent hip dysplasia requires a proper assessment of the degree of dysplasia and the surgery needed to redirect pelvic components to achieve suitable conditions for hip remodeling, and our mid- to long-term results showed very good outcomes when applying these principles using the llizarov technique.
\end{abstract}

Conclusão O tratamento da displasia do quadril na adolescência requer uma avaliação adequada do grau de displasia e a cirurgia necessária para reorientar os componentes pélvicos com o objetivo de alcançar condições adequadas para a remodelação do quadril. Nossos resultados de médio a longo prazo mostraram desfechos muito bons ao aplicarmos esses princípios usando a técnica de llizarov.

\section{Introdução}

Redirecionar a osteotomia pélvica tripla e outras osteotomias na região do quadril ganhou popularidade há muito tempo, com o objetivo de diminuir ou impedir maior progressão da osteoartrite displásica congênita do quadril, o que também apresenta bons resultados em curto, médio e longo prazos em termos de alívio dos sintomas para a maioria dos pacientes, mas uma proporção menor não apresenta melhora, e uma proporção menor ainda apresenta progressão dos sintomas, apesar da intervenção cirúrgica. ${ }^{1-12}$

Incongruência e sintomas osteoartríticos precoces das superfícies articulares são considerados por alguns autores preditores negativos para cirurgia de preservação articular. $^{9,13} \mathrm{O}$ ponto de vista oposto é o de que a osteotomia pélvica tripla pode ser uma alternativa à cirurgia de substituição articular precoce. ${ }^{12,14} \mathrm{O}$ aumento da área de contato entre a cabeça congruente e o acetábulo pode promover a remodelação da cartilagem degenerada. ${ }^{10}$ Alguns autores acreditam que a complexidade técnica e os potenciais corretivos estão correlacionados. ${ }^{8}$ No entanto, a osteotomia pélvica tripla é um método confiável para reconstruir a displasia acetabular residual e promover remodelação pélvica futura e contenção da cabeça femoral em pacientes jovens e adolescentes. ${ }^{15}$ Com mais de 25 anos de experiência usando a técnica de Ilizarov para reconstrução do quadril em crianças, relatamos nossos resultados de médio a longo prazo na reconstrução do quadril em adolescentes com quadris displásicos devido a displasia do desenvolvimento do quadril. 

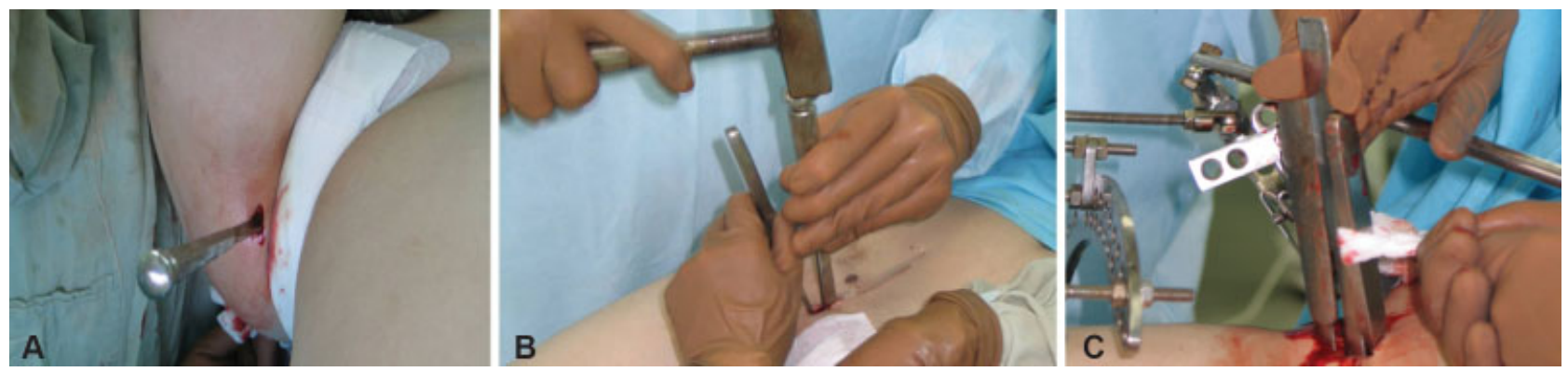

Fig. 1 Osteotomia tripla de Steel. ${ }^{18}$ (A) osteotomia do osso ísquio. (B) Osteotomia do osso púbico. (C) Osteotomia do osso ilíaco.

\section{Materiais e Métodos}

Entre 2002 e 2014, com aprovação do Conselho de Revisão Institucional (CRI) e do Comitê de Ética de nossa instituição, foram analisados os resultados do tratamento de 26 pacientes com coxartrose displásica. A idade média na intervenção foi de 14,7 anos (variação: 12-18 anos). A amostra continha 22 mulheres e 4 homens. 0 acompanhamento médio foi de 5,9 anos (variação: 3-13 anos). Nossos critérios de inclusão foram: 1) pacientes com idade $>12$ e $<19$ anos; 2) com grau I ou II para displasia do desenvolvimento do quadril (DDQ) de acordo com Tönnis et al; ${ }^{16} 3$ ) com graus II, III e IV de congruência articular de acordo com os critérios de Coleman; e 4) período de acompanhamento $\geq 3$ anos. Os critérios de exclusão foram: 1) pacientes com idade $<12$ e $>19$ anos; 2) com grau 0 e III para DDQ de acordo com Tönnis et al; ${ }^{16} 3$ ) com grau I de congruência articular de acordo com Coleman; e 4) e com quadris displásicos por outras causas que não a DDQ.

Histórico clínico e exame, além de radiografias pélvicas simples, foram utilizados para o diagnóstico pré-operatório e para acompanhar os resultados funcionais. O desfecho clínico foi avaliado pelos critérios de Merle d'Aubigné e Postel, ${ }^{17}$ e a melhora radiográfica, pelos critérios de Severin. A avaliação qualitativa da condição do quadril foi realizada de acordo com os critérios de Tönnis et al $^{16}$ e Coleman. As radiografias estudadas da articulação do quadril foram realizadas nas projeções anteroposterior e lateral antes da operação, durante o tratamento, e ao longo de todo o período de acompanhamento. Desenho manual sobre raios-X foi usado para avaliar os parâmetros radiográficos. Os seguintes parâmetros radiográficos foram calculados: índice da zona de carga (ZC), coeficiente acetabular (CA), ângulo cervicodiafisário (ACD), distância articulotrocantérica (DAT), ângulo centro-borda $(A C B)$ de Wiberg, ângulo central-vertical-anterior (CVA), e índice de migração (IM).

Todos os pacientes foram submetidos a reconstrução extraarticular do quadril com o aparelho de Ilizarov para correção da displasia acetabular, e foram realizadas osteotomias pélvicas como as de Carlioz et al $^{1}$ e Steel ${ }^{18}$ (- Fig. 1 e 2). Em seis casos, a osteotomia pélvica foi complementada com osteotomia periacetabular incompleta para alterar a forma do arco da cavidade. Em todo o estudo, com 26 pacientes, fizemos osteotomia pélvica. A correção da deformidade proximal do fêmur foi realizada em 22 casos, além da pélvica, cujos detalhes são os seguintes: em cinco casos fizemos osteotomia de destorção, em oito casos, osteotomia de desrotação do varo, em quatro casos, osteotomia de desrotação do valgo, e, em cinco casos, osteotomia transtrocantérica dupla (-Fig. 3). Nos quatro casos restantes, foram feitas apenas osteotomias pélvicas. $\mathrm{O}$ aparelho de Ilizarov foi utilizado para fixar os fragmentos osteotomizados. No decorrer do tratamento, foi realizado destracionamento de
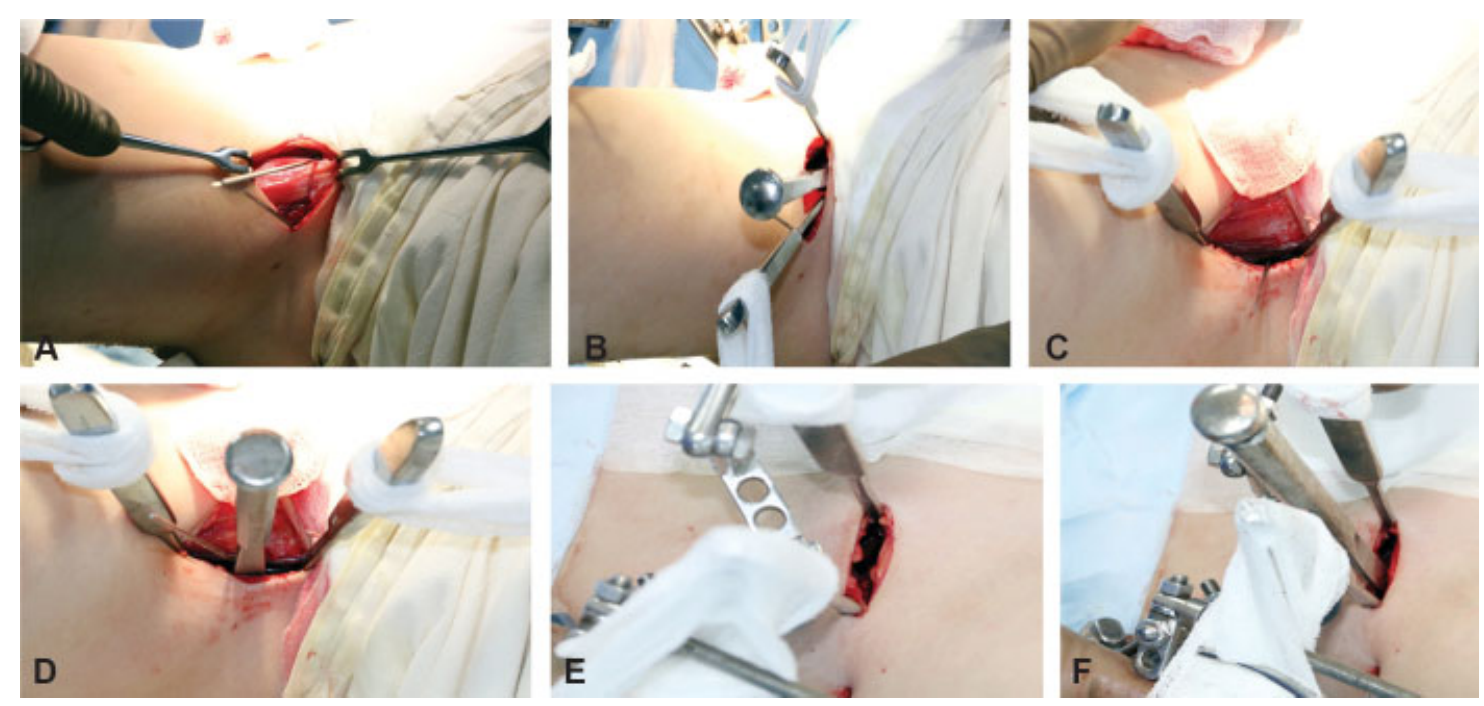

Fig. 2 Osteotomia de Carlioz et al. ${ }^{1}$ (A, B) Osteotomia do osso púbico. (C, D) Osteotomia do osso ísquio. (E, F) Osteotomia do osso ilíaco. 

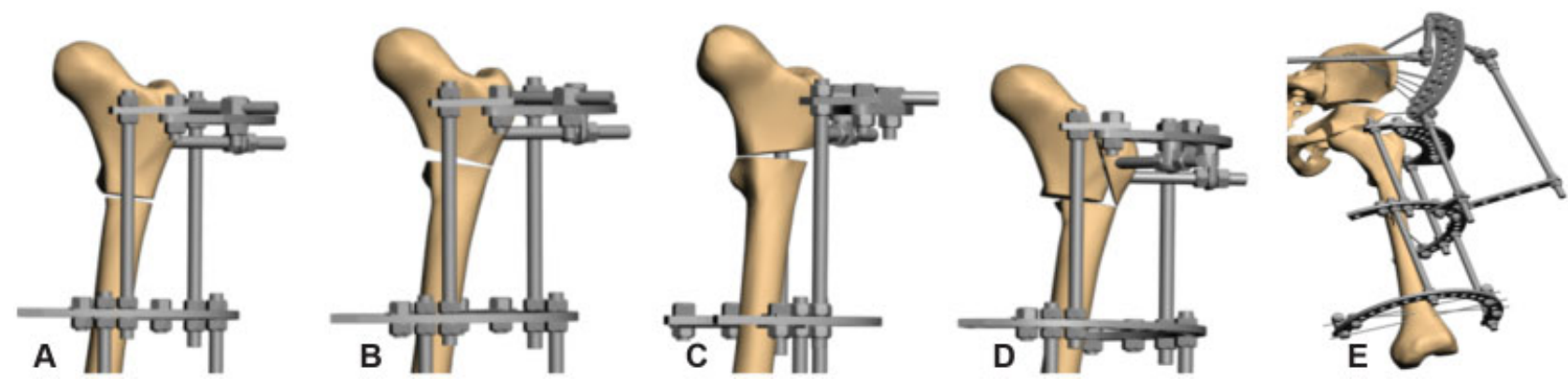

Fig. 3 Técnicas de reconstrução dos componentes femorais e pélvicos do quadril. (A) Osteotomia subtrocantérica de destorsão. (B) Osteotomia subtrocantérica de desrotação do valgo. (C) Osteotomia subtrocantérica de desrotação do varo. (D) Osteotomia transtrocantérica dupla. (E) osteotomia pélvica ligada à estrutura da armação.

apoio entre as superfícies articulares para proporcionar um modo de descompressão.

Os valores radiográficos foram analisados usando o programa Microsoft Excel 2007 (Microsoft Corp., Redmond WA, EUA). Os ângulos e outros parâmetros foram calculados como médias \pm desvios padrão, e o valor de $p$ da significância estatística foi calculado usando dispersão de dados, erro, e o teste $t$ de Student. Valores de $p<0,05$ foram considerados significativos. Os pacientes ou seus responsáveis deram consentimento para os fins do estudo, sem identificação pessoal.

\section{Técnica Cirúrgica}

Na sala de cirurgia, sob anestesia geral, 5 fios de Kirschner (fios k) olivados são passados pela asa ilíaca, pelo menos um deles na direção oposta, e outros 4 fios k são passados para a metáfise femoral distal. Todos os fios são então conectados ao aparelho de Ilizarov por arcos que são conectados por hastes ( - Fig. $\mathbf{3}$ ). Usando o método de Steel ${ }^{18}$ ( - Fig. 1a-c), realizamos incisão longitudinal de $3-4 \mathrm{~cm}$ lateralmente a partir da tuberosidade isquiática para osteotomizar o ísquio, uma incisão de $2-3 \mathrm{~cm}$ medial ao feixe neurovascular, e, abaixo do ligamento inguinal, realizamos osteotomia púbica e uma incisão de 3-4 cm acima da espinha ilíaca anterossuperior para realizar a osteotomia ilíaca. Após mobilizar os fragmentos em posição favorável para a cobertura da cabeça anterolateral, o fragmento acetabular é fixado à estrutura pélvica usando fios ou pinos de Schanz (-Fig. 4) na região supra-acetabular. Se a técnica de Carlioz et $\mathrm{al}^{1}$ for usada, uma incisão de $5 \mathrm{~cm}$ a $6 \mathrm{~cm}$ na superfície interna da coxa é feita $1 \mathrm{~cm}$ distal à prega inguinal, e, entre os músculos adutor longo e pectíneo, fazemos a dissecção romba para alcançar o osso púbico e realizar a osteotomia (-Fig. 2a,b). Depois, pela mesma incisão e entre os músculos adutor longo e magno, atingimos o osso ciático para realizar uma osteotomia transversal distal à coluna ciática ( $\mathbf{F i g}$. $\mathbf{2 c}$, d). A osteotomia do ílio e a cobertura acetabular são realizadas como na osteotomia de Steel. ${ }^{18}$ Se necessário, uma osteotomia periacetabular incompleta pode ser realizada por uma incisão de $5 \mathrm{~cm}$ a $6 \mathrm{~cm}$ ao longo do vinco inguinal entre as origens do sartório e do músculo tensor da fáscia lata. Deve-se tomar cuidado para não violar a cartilagem articular. $O$ fragmento supra-acetabular é então conectado à armação por meio de fios ou pinos de Schanz. A cirurgia corretiva no fêmur é realizada duas semanas após a osteotomia pélvica. Vale ressaltar que, no caso da técnica de Ilizarov, e quando as osteotomias pélvica e femoral são
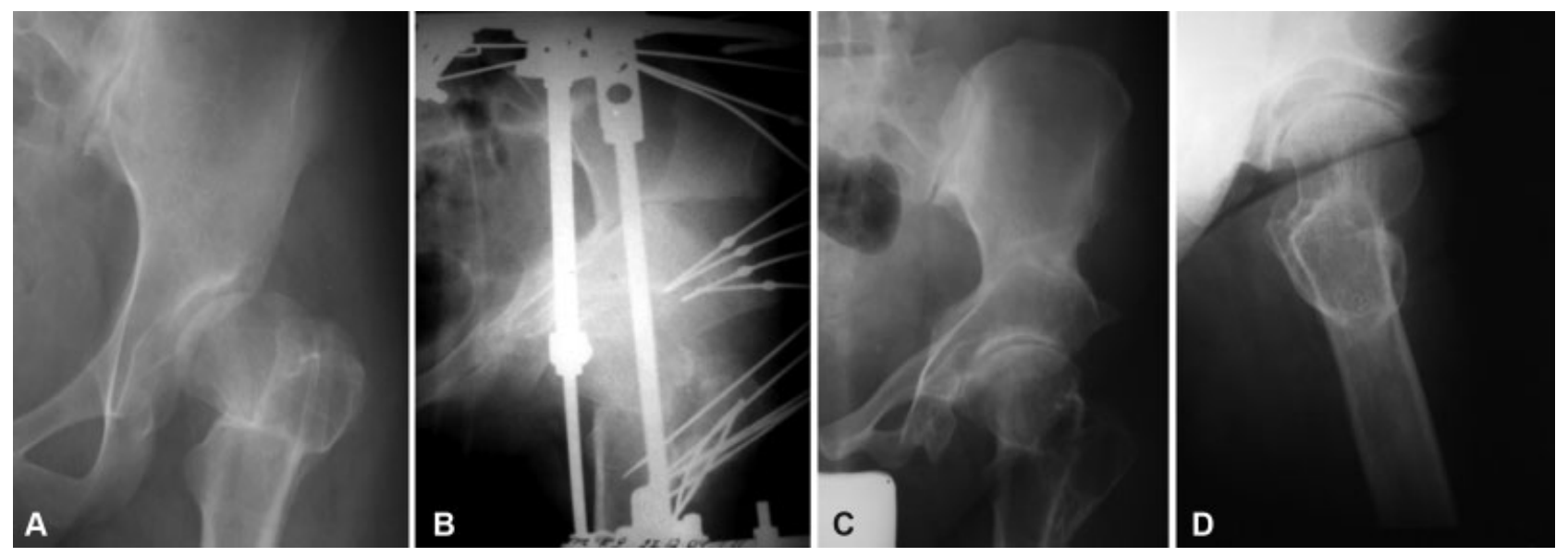

Fig. 4 Paciente do sexo feminino, de 16 anos, com coxartrose displásica esquerda. (A) Radiografia com osteoartrite de grau I, congruência de grau II de Coleman de superfícies articulares, índice da zona de carga (ZC) de $28^{\circ}$, coeficiente acetabular (CA) de 190, ângulo centro-borda (ACB) de $5^{\circ}$, ângulo central-vertical-anterior (CVA) de $5^{\circ}$, e índice de migração (IM) de 32\%. (B) Radiografia pélvica anteroposterior da reconstrução do quadril com redução fechada e com osteotomias pélvicas transtrocantéricas tripla e dupla. (C, D), Radiografia anteroposterior e lateral aos 12 anos de acompanhamento, com contenção da cabeça do fêmur, congruência de grau I de Coleman, ZC de $0^{\circ}$, CA de $210, A C B$ de $30^{\circ}$, ângulo CVA de $20^{\circ}$, e IM de $0 \%$. 
realizadas separadamente, elas se tornam intervenções relativamente simples e menos traumáticas. No entanto, a cirurgia simultânea é possível, mas, nesse caso, a duração e a agressividade da operação aumentam. Portanto, o tratamento em duas etapas é aceito em nosso centro. Ao realizar a cirurgia do quadril duas semanas após a osteotomia pélvica, o período total de tratamento com o equipamento não muda, pois é determinado principalmente pelo período de consolidação dos ossos pélvicos. Com relação ao grupo estudado, também é importante mencionar que, no caso de incongruência inicial e deformação dos componentes da articulação, uma reconstrução em dois estágios facilita significativamente a adaptação das superfícies articulares.

\section{Resultados}

A duração do tratamento foi de $76 \pm 2,2$ dias. A duração da reabilitação é de $9 \pm 1,5$ meses. Os resultados funcionais iniciais de acordo com o critério de Merle d'Aubigné e Postel ${ }^{17}$ foram: dor - 4,3 $\pm 0,05$ pontos; amplitude de movimento (ADM) - 3,6 $\pm 0,3$; e marcha $-4 \pm 0,15$ pontos. 0 principal sinal radiográfico em todas as articulações foi displasia acetabular e subluxação da cabeça femoral. A média do índice da ZC foi de $38,7^{\circ} \pm 2,72^{\circ}$, e o CA) foi de $162 \pm 6,8$. O ACB de Wiberg foi de $3^{\circ} \pm 0,2^{\circ}$, o ângulo CVA foi $10^{\circ} \pm 1,5^{\circ}$, e o IM foi de $38 \% \pm 4,5 \%$. Em todos os casos, houve uma anteversão excessiva do colo do fêmur $(41,6 \pm 1,24)$. Em 22 casos, foram observados vários tipos de deformidades proximais do fêmur, que foram corrigidas de acordo com suas características ( - Fig. 3). Em 8 casos, houve deformidade em valgo do pescoço, com ACD de $142,4^{\circ} \pm 1,44^{\circ}$. Em 9 casos, houve coxa vara definida, com ACD de $96,5^{\circ} \pm 2,344^{\circ}$, e encurtamento do pescoço, com DAT de $9,8 \pm 2,01 \mathrm{~mm}$. Em cinco casos, houve apenas desvio do pescoço no plano horizontal.

A distribuição das articulações pelo grau de deslocamento de acordo com Tönnis et a ${ }^{16}$ foi: grau I - 10 casos; grau II - 13 casos; e grau III -3 casos. O grau de coxartrose foi avaliado de acordo com Tönnis et al: ${ }^{16}$ grau I - 16 casos; e grau II -10 casos. O tipo de congruência das superfícies articulares foi avaliado de acordo com Coleman: grau II -7 casos; grau III - 8 casos; e grau IV - 11 casos.

Os resultados foram acompanhados por 3 a 13 anos. Os resultados funcionais de acordo com Merle d'Aubigné e Postel ${ }^{17}$ foram: dor $-4,7 \pm 0,1$ pontos; $\mathrm{ADM}-4,1 \pm 0,2$ pontos; e marcha $-4,6 \pm 0,1$ pontos. De acordo com os resultados radiográficos, o valor da $\mathrm{ZC}$ diminuiu para $8,2^{\circ} \pm 1,293^{\circ}$, e o CA amentou para $249 \pm 12,05$. A média do ACD foi de $115^{\circ} \pm 4^{\circ}$, e da DAT, $8,5 \pm 1,5 \mathrm{~mm}$. Os índices médios de redução melhoraram com ACB de Wiberg de $28^{\circ} \pm 1,6^{\circ}$, ângulo CVA de $26^{\circ} \pm 1,5^{\circ}$, e IM de $12,6 \pm 1,5$. Houve diferença significativa entre todos os valores pré- e pós-operatórios (-Tabela 1). Os achados radiográficos segundo os critérios de Severin foram: grau Ila - 14 casos; grau IIb - 8 casos; e grau III - 4 casos. De acordo com os critérios de Coleman, eles foram: grau I - 6 casos; grau II - 5 casos; grau III - 12 casos; e grau IV - 3 casos. A distribuição da articulação segundo Tönnis et al $^{16}$ foi: grau I - 17 articulações; grau II - 8 articulações; e grau III - 1 articulação. Houve melhora na congruência das superfícies articulares, e, no acompanhamento
Tabela 1 Parâmetros radiográficos no pré-operatório, no pósoperatório, e resultados do último acompanhamento

\begin{tabular}{|l|l|l|l|}
\hline \multirow{2}{*}{ Parâmetros } & \multicolumn{3}{|l|}{ Intervalos de tratamento } \\
\cline { 2 - 4 } & Pré-operatório & $\begin{array}{l}\text { Após a } \\
\text { remoção } \\
\text { da armação }\end{array}$ & $\begin{array}{l}\text { Último } \\
\text { acompanhamento }\end{array}$ \\
\hline ACD ( $\left.{ }^{\circ}\right)$ & $118,5 \pm 3,9$ & $121 \pm 1,0^{*}$ & $\begin{array}{l}116 \pm 1,0^{*} \\
p<0,05\end{array}$ \\
\hline AA ( $\left.{ }^{\circ}\right)$ & $41,6 \pm 1,6$ & $\begin{array}{l}15 \pm 0,8^{*} \\
p<0,001\end{array}$ & $16 \pm 0,8$ \\
\hline $\begin{array}{l}\text { DAT } \\
(\mathrm{mm})\end{array}$ & $7,2 \pm 2,8$ & $\begin{array}{l}13,7 \pm 0,3^{*} \\
p<0,05\end{array}$ & $11,5 \pm 0,4^{*}$ \\
\hline ZC & $39 \pm 1,6$ & $\begin{array}{l}9 \pm 0,7^{*} \\
p<0,001\end{array}$ & $8,2 \pm 0,8$ \\
\hline CA & $162 \pm 1,9$ & $\begin{array}{l}190 \pm 7,5^{*} \\
p<0,01\end{array}$ & $249 \pm 6,6$ \\
& & $\begin{array}{l}30,5 \pm 1,4^{*} \\
p<0,001\end{array}$ & $28 \pm 1,8$ \\
\hline ACB ( $\left.{ }^{\circ}\right)$ & $3 \pm 0,8$ & $\begin{array}{l}8,5 \pm 1,5^{*} \\
p<0,001\end{array}$ & $12,6 \pm 1,8$ \\
\hline IM (\%) & $38 \pm 1,5$ & $\begin{array}{l}27 \pm 0,8^{*} \\
p<0,01\end{array}$ & $26 \pm 0,9$ \\
\hline CVA ( $\left.{ }^{\circ}\right)$ & $10 \pm 0,7$ & & \\
\hline
\end{tabular}

Abreviaturas: AA, ângulo epifisário de Alsberg; $A C B$, ângulo centroborda; $A C D$, ângulo cervicodiafisário; $C A$, coeficiente acetabular; $C V A$, ângulo central-vertical-anterior; DAT, distância articulotrocantérica; IM, índice de migração; ZC, zona de carga.

Nota: *Valor de $p$ estatisticamente significativo.

final, o grau de artrose permaneceu inalterado em 20 casos, reduzido em 4 articulações, e progrediu 1 grau em 2 articulações. No geral, os desfechos foram bons em 14 pacientes (54\%), razoáveis em 10 pacientes (34,5\%), e ruins em 2 (11,5\%), o que significa resultados positivos, com interrupção da progressão da osteoartrite em 24 pacientes (88,5\%) (-Figs. 4 e 5 ).

\section{Discussão}

Na displasia do quadril, o componente acetabular é geralmente afetado, assim como o femoral, mas em menor grau. No acetábulo raso, a cabeça do fêmur é lateralizada e deslocada com proporções anatômicas anormais dos componentes do quadril, perturbação da biomecânica, e contratura muscular e ligamentar periarticular. ${ }^{2,19}$ Muitas osteotomias de reorientação pélvica para displasia do quadril sintomático podem fornecer resultados satisfatórios em longo prazo. ${ }^{5,16,18-22} \mathrm{O}$ aumento da área de contato entre a cabeça congruente e o acetábulo pode promover a remodelação da cartilagem degenerada. ${ }^{10}$ A osteotomia pélvica, mesmo no segundo estágio da coxartrose, pode impedir que a coxartrose se desenvolva ainda mais, ${ }^{12,23}$ e até mesmo promover sua regressão. ${ }^{7}$ Diferentes técnicas de osteotomia pélvica de redirecionamento foram estudadas e comparadas, ${ }^{24}$ incluindo a osteotomia periacetabular de Bernese ${ }^{25}$ sendo que algumas permitem rotação em direção indesejada e resultam em desfechos piores. Na comparação das osteotomias de Ganz, Carlioz et al ${ }^{1}$ e Tönnis et al, ${ }^{16}$ a osteotomia de Carlioz et al $^{1}$ proporcionou menos movimento, com deslocamento previsível dos fragmentos de osteotomia nas direções propostas. As de Tönnis et al $^{16}$ e Ganz 


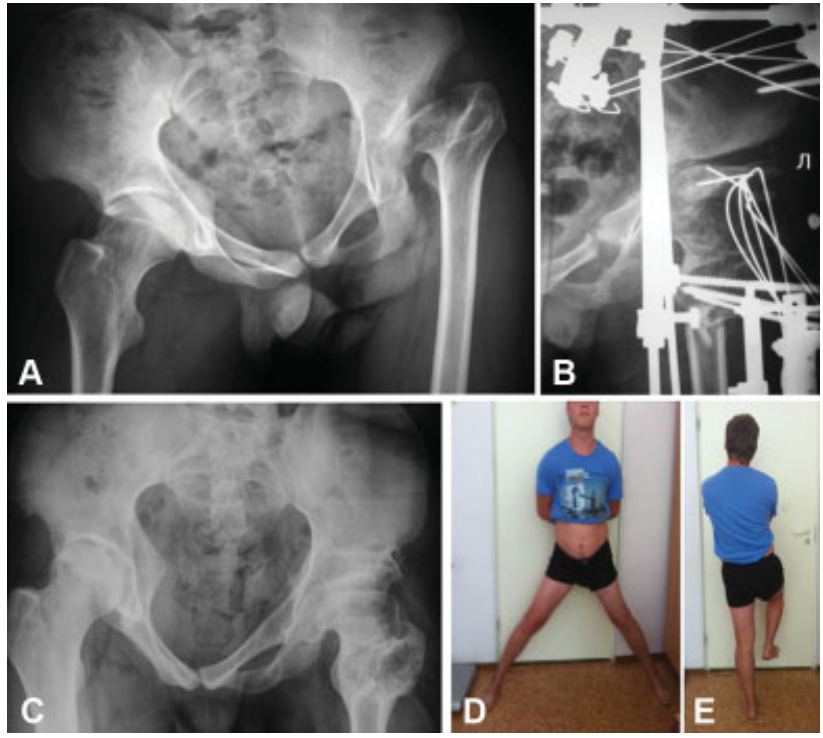

Fig. 5 Paciente de 19 anos, do sexo masculino, com coxartrose displásica esquerda. (A) Radiografia com osteoartrite de grau I e luxação de grau II, incongruência de grau IV de Coleman, ZC de $43^{\circ}$, CA de 160, ACB $0^{\circ}$, ângulo CVA de $0^{\circ}$, e IM de $48 \%$. (B) Radiografia pélvica anteroposterior da reconstrução do quadril, com redução fechada e com osteotomias pélvicas transtrocantéricas tripla e dupla. (C) Radiografia anteroposterior com 4 anos de acompanhamento, com redução, ZC de $8^{\circ}$, CA de 210 , ACB de $20^{\circ}$, ângulo CVA de $22^{\circ}$, e IM de 16\%. (D, E) Abdução e teste negativo de Trendelenburg.

permitiram movimentos sem restrições em diferentes direções. ${ }^{24}$ A osteotomia de Carlioz et al $^{1}$ foi usada em conjunto com a de Steel ${ }^{18}$ neste estudo. Pacientes jovens com quadril displásico devido a DDQ podem se beneficiar das osteotomias intertrocantéricas e pélvicas para adiar ou eliminar a necessidade de artroplastia total do quadril (ATQ). 2,4,6,12,14 $\mathrm{O}$ uso da técnica de Ilizarov para redução da displasia do quadril e a fixação de múltiplas osteotomias ao mesmo tempo, sem a necessidade de redução aberta e encurtamento do fêmur, mostraram bom resultado, com menor prevalência de necrose avascular. $^{26}$

Acreditamos que a idade do paciente na operação desempenha um papel importante no desfecho, com pacientes que completaram sua maturidade esquelética obtendo desfechos menos favoráveis. Alguns estudos ${ }^{2,4,27}$ foram realizados em amostras de faixa etária mais ampla, com alguns pacientes tendo necessitado conversão para ATQ mais cedo. Okano et $\mathrm{al}^{14}$ demonstraram progressão em 11 (25\%) pacientes da sua amostra, mas a média de idade deles era de 43,4 anos (variação de 30 a 59 anos). Um resultado médio foi observado no estudo de Böhm et al, ${ }^{6}$ com pacientes com idade média no momento da operação de 24,8 anos (variação de 19 a 35 anos), que tiveram resultado médio, com diminuição da coxartrose em 11 quadris, nenhuma mudança em 17, e piora em 5 quadris. Em contraste com esses resultados, nossa amostra incluiu pacientes adolescentes (12 a 18 anos), e resultou em $88,5 \%$ de resultados positivos, e piora em apenas 2 casos (11,5\%); resultados semelhantes foram obtidos por outros autores ${ }^{3,18}$ com estudos em pacientes jovens.

Com relação ao grau de osteoartrite no pré-operatório, em estudo realizado por Van Hellemondt et al $^{11}$ (embora seus fatores negativos significativos para bons resultados em longo prazo fossem a presença de alterações osteoartríticas e um escore clínico razoável ou ruim no pré-operatório), os autores relataram seus resultados com osteotomia pélvica tripla de 48 quadris, em que 42 pacientes (88\%) com acompanhamento em longo prazo de 15 anos não apresentaram progressão da osteoartrite, e com bons ou excelentes resultados clínicos em 27 (64\%) deles. Curiosamente, Johnsen et $\mathrm{al}^{22}$ descobriram que, entre a população Sámi da Noruega, um quadril com DDQ tem baixo valor preditivo de osteoartrite radiográfica concomitante. Assim, os índices radiográficos não podem prever a taxa na qual a articulação do quadril desenvolverá osteoartrite; no entanto, acreditamos que não há contraindicação per se para realizar reorientação de osteotomias pélvicas em pacientes com alterações radiográficas displásicas. No que diz respeito à escolha da osteotomia, preferimos a técnica de Carlioz et $\mathrm{al}^{1}$ se for necessária uma transposição considerável do acetábulo.

No que diz respeito ao método de fixação após a osteotomia, é mais provável que os métodos convencionais de fixação sejam fios $\mathrm{k}$ robustos ou pinos e placas, e alguns autores relataram maior incidência de não união óssea, que está associada à insatisfação do paciente. ${ }^{4,27,28}$ Em nosso estudo, não encontramos nenhum caso de não união, provavelmente por causa da relativa estabilidade da estrutura. No entanto, se essa possibilidade se concretizasse, ainda poderíamos modificar a estrutura e adicionar ou remover fios para dinamizar os fragmentos ósseos, conforme necessário. Outras complicações, como infecção, sangramento e dor pós-operatória podem ser incômodas. Trousdale et $\mathrm{al}^{4}$ relataram dor relacionada ao equipamento, o que levou à sua remoção em 9 pacientes (21\%) de sua série. Em relação às nossas complicações, tivemos dois casos de infecção no trajeto de fios e/ou pinos (ITFP), que foram tratados com cefazolina intravenosa (IV) e troca frequente de curativos. Também tivemos 2 casos com rápido desenvolvimento de osteoartrite durante os primeiros 2 anos de acompanhamento: 1 paciente apresentou correção excessiva da cabeça femoral, mas isso foi refutado por Klein et al ${ }^{15}$ no estudo em que descobriram que a sobrecorreção nos 3 planos em 33 $(80,5 \%)$ quadris não apresentou diferença significativa entre estes e quadris com parâmetros normais. Acreditamos que a causa foi o impacto femoroacetabular e o estreitamento do espaço articular, como aparecia na radiografia. 0 outro paciente teve déficit de cobertura da cabeça femoral pela osteotomia, que provavelmente desempenhou um papel na progressão da osteoartrite. Em nossa opinião, as vantagens de nossa técnica se devem principalmente ao uso do aparelho de Ilizarov, com grande versatilidade de correção e movimento dos fragmentos acetabulares, e que também é menos invasivo e resulta em menos perda de sangue, o que pode estar associado a um melhor desfecho. ${ }^{3,29} \mathrm{O}$ uso da técnica de Ilizarov também permite reabilitação precoce, com pacientes capazes de caminhar no segundo dia de pós-operatório.

O uso da técnica de Ilizarov para correção e alongamento das deformidades isquêmicas do quadril tem sido apresentado com bons resultados em médio prazo. ${ }^{30}$ No entanto, alguns pacientes podem se sentir desconfortáveis com a 
estrutura volumosa, mas com o tempo eles se acostumam e se sentem confortáveis, focados no tratamento. Outra limitação é que o uso da técnica de Ilizarov requer experiência do cirurgião, a qual demanda algum tempo para ser adquirida.

\section{Conclusão}

A aplicação da técnica de Ilizarov para cirurgia reconstrutiva pélvica devido a displasia congênita do quadril em adolescentes provou ser um método bem-sucedido e confiável. A técnica pode ser uma alternativa para a substituição articular em adolescentes e adultos jovens, e proporciona funcionalidade suficiente à articulação afetada. No geral, nossos resultados mostraram desfechos bons em 14 pacientes (54\%), razoáveis em 10 pacientes (34,5\%), e ruins em 2 $(11,5 \%)$ pacientes, o que significa que um desfecho positivo, com a interrupção da progressão da osteoartrite, foi observado em 24 pacientes $(88,5 \%)$.

\section{Conflito de Interesses}

Os autores declaram não haver conflito de interesses.

\section{Referências}

1 Carlioz H, Khouri N, Hulin P. [Triple juxtacotyloid osteotomy]. Rev Chir Orthop Repar Appar Mot 1982;68(07):497-501

2 Haverkamp D, Marti RK. Intertrochanteric osteotomy combined with acetabular shelfplasty in young patients with severe deformity of the femoral head and secondary osteoarthritis. A longterm follow-up study. J Bone Joint Surg Br 2005;87(01):25-31

3 Lehman WB, Mohaideen A, Madan S, Atar D, Feldman DS, Scher D. Surgical technique for an 'almost' percutaneous triple pelvic osteotomy for femoral head coverage in children 6-14 years of age. J Pediatr Orthop B 2004;13(01):57-62

4 Trousdale RT, Ekkernkamp A, Ganz R, Wallrichs SL. Periacetabular and intertrochanteric osteotomy for the treatment of osteoarthrosis in dysplastic hips. J Bone Joint Surg Am 1995;77(01):73-85

5 de Kleuver M, Kooijman MA, Pavlov PW, Veth RP. Triple osteotomy of the pelvis for acetabular dysplasia: results at 8 to 15 years. J Bone Joint Surg Br 1997;79(02):225-229

6 Böhm P, Klinger HM, Küsswetter W. The Salter innominate osteotomy for the treatment of developmental dysplasia of the hip in young adults. Arch Orthop Trauma Surg 1999;119(3-4):186-189

7 Sen C, Sener N, Tozun IR, Boynuk B. Polygonal triple (Kotz) osteotomy in the treatment of acetabular dysplasia: 17 patients (19 hips) with 4-9 years of follow-up. Acta Orthop Scand 2003;74(02):127-132

8 Gillingham BL, Sanchez AA, Wenger DR. Pelvic osteotomies for the treatment of hip dysplasia in children and young adults. J Am Acad Orthop Surg 1999;7(05):325-337

9 Søballe K. Pelvic osteotomy for acetabular dysplasia. Acta Orthop Scand 2003;74(02):117-118

10 Shimogaki K, Yasunaga Y, Ochi M. A histological study of articular cartilage after rotational acetabular osteotomy for hip dysplasia. J Bone Joint Surg Br 2005;87(07):1019-1023

11 van Hellemondt GG, Sonneveld H, Schreuder MH, Kooijman MA, de Kleuver M. Triple osteotomy of the pelvis for acetabular dysplasia: results at a mean follow-up of 15 years. J Bone Joint Surg Br 2005;87(07):911-915
12 Janssen D, Kalchschmidt K, Katthagen BD. Triple pelvic osteotomy as treatment for osteoarthritis secondary to developmental dysplasia of the hip. Int Orthop 2009;33(06):1555-1559

13 Yasunaga Y, Ochi M, Terayama H, Tanaka R, Yamasaki T, Ishii Y. Rotational acetabular osteotomy for advanced osteoarthritis secondary to dysplasia of the hip. J Bone Joint Surg Am 2006;88(09): 1915-1919

14 Okano K, Enomoto H, Osaki M, Shindo H. Rotational acetabular osteotomy for advanced osteoarthritis secondary to developmental dysplasia of the hip. J Bone Joint Surg Br 2008;90(01):23-26

15 Klein C, Fontanarosa A, Khouri N, et al. Anterior and lateral overcoverage after triple pelvic osteotomy in childhood for developmental dislocation of the hip with acetabular dysplasia: Frequency, features, and medium-term clinical impact. Orthop Traumatol Surg Res 2018;104(03):383-387

16 Tönnis D, Arning A, Bloch M, Heinecke A, Kalchschmidt K. Triple pelvic osteotomy. J Pediatr Orthop B 1994;3:54-67

17 d'Aubigné RM, Postel M. The classic: functional results of hip arthroplasty with acrylic prosthesis. 1954. Clin Orthop Relat Res 2009;467(01):7-27

18 Steel HH. Triple osteotomy of the innominate bone. A procedure to accomplish coverage of the dislocated or subluxated femoral head in the older patient. Clin Orthop Relat Res 1977;(122):116-127

19 Millis MB, Kim YJ. Rationale of osteotomy and related procedures for hip preservation: a review. Clin Orthop Relat Res 2002;(405): 108-121

20 Millis MB, Poss R, Murphy SB. Osteotomies of the hip in the prevention and treatment of osteoarthritis. Instr Course Lect 1992;41:145-154

21 Tönnis D. Congenital dysplasia and dislocation of the hip. Berlin: Springer-Verlag; 1987

22 Johnsen K, Goll R, Reikerås O. Acetabular dysplasia as an aetiological factor in development of hip osteoarthritis. Int Orthop 2009; 33(03):653-657

23 Okano K, Enomoto H, Osaki M, Shindo H. Outcome of rotational acetabular osteotomy for early hip osteoarthritis secondary to dysplasia related to femoral head shape: 49 hips followed for 10 17 years. Acta Orthop 2008;79(01):12-17

24 Aminian A, Mahar A, Yassir W, Newton P, Wenger D. Freedom of acetabular fragment rotation following three surgical techniques for correction of congenital deformities of the hip. J Pediatr Orthop 2005;25(01):10-13

25 Leunig M, Siebenrock KA, Ganz R. Rationale of periacetabular osteotomy and background work. Instr Course Lect 2001; 50:229-238

26 Teplenky M, Mekki W. Reduction by pelvic external fixator followed by innominate and derotational femoral osteotomies for late presenting bilateral developmental dysplasia of the hip. J Orthop Trauma Rehabil. 2018;25:76-81

27 Hailer NP, Soykaner L, Ackermann H, Rittmeister M. Triple osteotomy of the pelvis for acetabular dysplasia: age at operation and the incidence of nonunions and other complications influence outcome. J Bone Joint Surg Br 2005;87(12):1622-1626

28 Tschauner C, Sylkin A, Hofmann S, Graf R. Painful nonunion after triple pelvic osteotomy. Report of five cases. J Bone Joint Surg Br 2003;85(07):953-955

29 Wall EJ, Kolata R, Roy DR, Mehlman CT, Crawford AH. Endoscopic pelvic osteotomy for the treatment of hip dysplasia. J Am Acad Orthop Surg 2001;9(03):150-156

30 Teplenky M, Mekki W. Pertrochanteric osteotomy and distraction femoral neck lengthening for treatment of proximal hip ischemic deformities in children. J Child Orthop 2016;10(01):31-39 